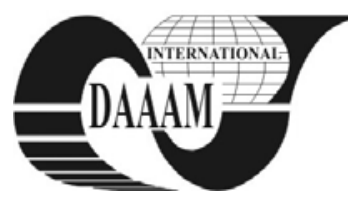

Annals of DAAAM for 2011 \& Proceedings of the 22nd International DAAAM Symposium, Volume 22, No. 1, ISSN 1726-9679 ISBN 978-3-901509-83-4, Editor B. Katalinic, Published by DAAAM International, Vienna, Austria, EU, 2011 Make Harmony between Technology and Nature, and Your Mind will Fly Free as a Bird Annals \& Proceedings of DAAAM International 2011

\title{
DYNAMIC BEHAVIOURAL DECISION SYSTEMS
}

\author{
BADESCU, A[drian] V[ictor] \& CRISTEA, R[adu] N[icolae]
}

\begin{abstract}
This paper explains mathematically the concept of investment agent's bounded rationality to bring a new perspective on the use of threats as means of analyzing competition. The paper thus blends together two approaches: strong efficiency economics and behavioral economics. Further we construct a dynamic situation and considering a two step duopoly we develop a new type of equilibrium - a fuzzy equilibrium - that is better suited for real decision situations.

Key words: fuzzy control theory, game theory, acquisition strategies
\end{abstract}

\section{INTRODUCTION}

The theory of efficient market hypothesis presented by Beechey (2000) (strong form efficiency) relies only on the current price of an asset to describe its overall performance. Given this fact and considering commonly used investment assessment tools like M.A.C.D. (i.e. moving average convergence/divergence) we are able to extrapolate and thus theorize that most of the information defining/representing a stock asset can rely only on the quantity that a investor has just paid in order to acquire it considering that quantity of acquisition determines demand and thus in most of the cases the future price of any good on any market.

Bearing in mind the above approach this article uses fuzzy theory to interpret mathematically (as close as possible to human logic) the bounded rationality of economic agents (i.e. stock market investors) a theory launched by Herbert Simon (1957) and known today as behavioral economics. This approach ensures flexibility and using fuzzy logic and control theory we discover a new way of using financial market communication between investors. In order to illustrate mathematically such a thing we have built a similar situation to a Stackelberg duopoly in which we will develop a new type of economic equilibrium - an approach that we consider defines the investor's logic in dynamic decision situations in a more adequate manner than the classical Nash approach.

On the financial market there has always been a difficulty in obtaining an interconnected outcome from the interactions among a group of self-interested individuals referred hereafter as agents. There are many ways to describe interactions between agents starting with the most common ones that entail a process of infinitely or finitely repeated interaction between them (described by Perdo Dal in 2005), but also fictitious play (described by Brown 1951) or the tit-for-tat strategy where we follow the principal and follower formula of strategy games. Please note that we are able to find these methods being applied in solving applications in economics, marketing, political science, production, biology and engineering.

Although all of the above mentioned approaches deal with the problem of dynamically modeling human rational behavior (Archer and Tritter 2000) they all take into account the hypothesis according to which an agent's cooperation or participation to the economic market is limited to a simple binary choice. Given this fact all the models suffer from a lack of interpretation of the "surrounding reality", as in the real world interaction between agents is not binary as Diamond (2010) argues, but rather continuous. This can be easily sustained if we consider the classic example of tit-for-tat where if agents were to participate to the market in a rather continuous manner forming their decisions based on different signal strengths that they perceive as to their own understanding than the outcome will definitely be a different one, as the play will develop in a more natural and human manner.

In order to sustain such an approach we propose the following idea: where agents view signals from other agents in a rather continuous manner we can obtain outcomes that are directly connected with on one hand the strength of the signal emitted and on the other with the perception of the received signal in this respect.

Such an approach can be used to allow a machine to take into the account of automated decisions: human behavior.

\section{THE FUZZY APPROACH}

In this context we assume that the agents use the following heuristics in order to better guide their decisions: the agent observes the signal, classifies the level of strength that it represents and makes a decision using the following guidelines:

As the signal strength received decreases so does the agent's involvement on the market.

As the signal strength received increases so does the agent's involvement on the market.

In order to implement mathematically such an approach it is necessary to use control theory in order to dynamically be able to establish a link between the fuzzy inputs given as signals that are relative in nature (signals that are interpreted by corresponding agents and that in fact represent their subrational and uncertain understanding of the things around them) and the output that in this case represents a investment decision within the financial market.

In respect to the above there are allot of methods for implementing a control system in order to be able to obtain the expected results and many of them have been summarized by Timothy (2010). The newest approach uses logic in order to ascertain the result. In other words the method uses previous experience and also logical rules like "IF case THEN outcome". Furthermore, the logic based method is actually similar to the commonly used experimental method because it also defines a table of inputs and corresponding output values where we use fuzzy values instead of having crisp numeric values as input and output variables.

After fuzzy reasoning in order for the result to be translated into a crisp value and thus make it comparable with other decision factors we use defuzzification which represents an inverse transformation that maps the output from the fuzzy domain back into the crisp domain. To accomplish this we use the Center-of-Area (C-o-A) method, often referred to as the Center-of-Gravity (C.O.G.) method because it computes the centroid of the composite area representing the output fuzzy term. In other words if we assume that we use a fuzzy triangle 
representation and the output is somewhere in between any two fuzzy numbers the area that we refer to will be the by dimensional space delimited by the combination of the two adjacent triangles.

\section{NUMERIC EXAMPLE}

For the following application we consider a start budget of EUR 20,000. The investment agent establishes a volume (denoted q) of 104 shares of IBM (International Business Machines Corp.) that are to be acquired given the price per share ratio of EUR 185.18.

All the other agents on the market (i.e. competing agents) consider safe ground when the investment agent acquires a volume of shares that is 80 or less. In this case the opposing agent will not let itself be influenced by the decisions of the investment agent. We also know that once the investment agent acquires 120 of shares the threat intensity perceived by the opposing agents will max out. We also know that between the safe ground and the max out interval the agents manifest a normal human reaction. In other words, an agent's behavior is somehow relaxed at first and rapidly transforms into an accelerated-strained one. As the influence shock diminishes the player returns to a neutral position. Please note that to reach such a conclusion we have reconstructed for IBM a similar statistical procedure as the one presented by Diamond (2008). Further we construct two separate input functions which will describe at best the given signal level, for our case. We denote by $\mathrm{a}_{1}(\mathrm{x})$ the preference function concerning a low level of threat and by $\mathrm{a}_{2}(\mathrm{x})$ the preference function for a high threat level:

$$
\mathbf{a}_{1}(x)=\left\{\begin{array}{ccc}
1 & \text { if } & x \leq 80 \\
1-2\left(\frac{x-80}{40}\right)^{2} & \text { if } & 80<x \leq 100 \\
2\left(\frac{120-x}{40}\right)^{2} & \text { if } & 100<x \leq 120 \\
0 & \text { if } & x>120
\end{array}\right.
$$
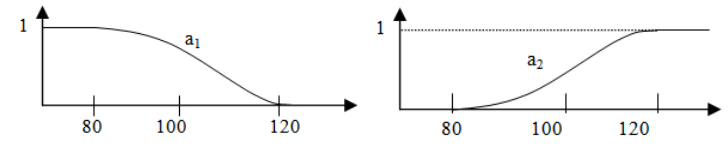

Fig 1. Membership function

$\mathbf{a}_{2}(x)=\left\{\begin{array}{ccc}0 & \text { if } & x \leq 80 \\ 2\left(\frac{x-80}{40}\right)^{2} & \text { if } & 80<x \leq 100 \\ 1-2\left(\frac{120-x}{40}\right)^{2} & \text { if } & 100<x \leq 120 \\ 1 & \text { if } & x>120\end{array}\right.$

Thus we obtain: $\mathrm{a}_{1}(104)=0.32$ and $\mathrm{a}_{2}(104)=0.68$.

In order to establish the level of the agent's involvement in the game we first determine the mapping function in respect to the opposing agent.

First, we will construct the preference relations for the noninvolvement and the involvement strategy, functions that reflect the agent's behavior as a response to the actions undertaken by the opposing agent that is in this case the investment agent. The functions are different from those which describe the level of threat, because there is always a difference between signal's strength and the behavior determined by it:

$$
\begin{aligned}
& \mathrm{b}_{1}(\mathrm{y})=1-4\left(\frac{\mathrm{y}-80}{80}\right)^{2} \text { if } 80 \leq \mathrm{y} \leq 120 ; \\
& \mathrm{b}_{2}(\mathrm{y})=4\left(\frac{\mathrm{y}-80}{80}\right)^{2} \text { if } 80 \leq \mathrm{y} \leq 120
\end{aligned}
$$

Using the C.O.G. approach we determine the aggregated values and the fuzzy centroid value given by the two above mentioned preference relations:

$$
\begin{aligned}
& \mathrm{V}_{1}=\int_{80}^{120} 1-4\left(\frac{\mathrm{x}-80}{80}\right)^{2} d x=26.66 ; \mathrm{V}_{2}=13.33 \\
& \mathrm{C}_{1}=\frac{\int_{80}^{120} x\left[1-4\left(\frac{\mathrm{x}-80}{80}\right)^{2}\right] d x}{\int_{80}^{120} 1-4\left(\frac{\mathrm{x}-80}{80}\right)^{2} d x}=95 ; \mathrm{C}_{2}=110
\end{aligned}
$$

The mapping function $\mathrm{F}(\mathrm{x})$ has the value 0.452 . Thus, by analyzing $\lim _{x \rightarrow \min } F(x)=c_{1}$ and $\lim _{x \rightarrow \max } F(x)=c_{2}$ the agent's noninvolvement intention is about $16.6 \%$. Also the level of intensity as a response towards investment agent that is perceived by the opposing agent amounts to $83.4 \%$.

Knowing the preference aggregation function concerning the perceived response, we are able to determine the acquisition volume chosen by the opposing agent as a response towards investment agent (the prior established volume of 104). By using $q=I \cdot \max$, we obtain $q=0.83 \cdot 120=99.6$ that represents the volume intended to be acquired. This represents the game's fuzzy equilibrium in the situation in which the opposing agent organizes its acquisition based solely on the threats perceived from the other market players.

\section{CONCLUSION}

Through this research, we are trying to develop a complex and detailed financial computer simulation meant to describe, analyze and thus forecast player's behavior based mainly on the conditions found in the market environment. At this particular stage, we have already designed and implemented into the software package new ways to mathematically illustrate and at least partially use human uncertain or rationality bounded behavior observed in an economic-financial environment in order to append these to the classic, cost minimizing while output maximizing, schemes.

Considering our approach, its main limitations come from out assertion of a normal human reaction and our approach of continuous interaction between agents which can differ. In order to automate such a process it is necessary to take into account all possible types of reactions.

Furthermore, using solely such a process will not render any specific results but if we are to implement it on a large scale, into a decision algorithm we will be able, to take into account how the market will respond to a acquisition and thus be able to influence the future distribution of a stock portfolio.

\section{ACKNOWLEDGEMENTS}

The paper represents a portion of the results obtained in the ongoing research Grant 1805, contract 774/2008, financed by the I.D.E.I. Programme PN. II with the title: "Exploratory research concerning the development of an intelligent system meant to optimize financial decisions"

\section{REFERENCES}

Diamond P. \& Vartiainen H. (2008) Behavioral Economics and Its Applications, Princeton University Press, 0691122849

Halpen J.Y. (2003) Reasoning About Uncertainty, MIT Press, 978-0262582599, USA

Kin K. L. \& Shouyang W (2010) Fuzzy Portfolio Optimization Springer

Klir, G. (1997), Fuzzy Set Theory: Foundations And Applications, Upper Saddle River, NJ : Prentice Hall,

Papayoanou P (2010) Game Theory for Business, Probabilistic Publishing 